\title{
Hadronic resonance production measured with the ALICE detector
}

\author{
Ajay Kumar Dash (For the ALICE Collaboration)*i \\ Instituto de Fisica Gleb Wataghin - UNICAMP, Brazil \\ E-mail: adashecern.ch
}

\begin{abstract}
Hadronic resonances serve as a unique tool to study the properties of hot and dense matter produced in heavy-ion collisions. These properties can be studied by measuring the ratios of hadronic resonance yields to the yields of longer-lived hadrons which can be used to investigate the rescattering effects and the chemical freeze-out temperature. Resonance measurements in pp and $\mathrm{p}-\mathrm{Pb}$ collisions provide a necessary baseline for heavy-ion data and help to disentangle the initialstate effects from medium-induced effects. The ALICE Collaboration has measured resonances such as, $\mathrm{K}^{*}(892)^{0}$ and $\phi(1020)$ in $\mathrm{pp}, \mathrm{p}-\mathrm{Pb}$, and $\mathrm{Pb}-\mathrm{Pb}$ collisions at the LHC energies. These resonances are reconstructed via their hadronic decay channel in a wide momentum range at midrapidity. In this work, we present recent results on the transverse momentum spectra, mean transverse momentum, ratios of resonance production relative to that of long-lived hadrons.
\end{abstract}

7th International Conference on Physics and Astrophysics of Quark Gluon Plasma

1-5 February, 2015

Kolkata, India

\footnotetext{
* Speaker.

${ }^{\dagger}$ Now at National Institute of Science Education and Research, Jatni, INDIA-752050.
} 


\section{Introduction}

Resonances serve as useful probes that allow the characteristics of heavy-ion collisions to be studied at different stages of their evolution. Several observables are of interest, including the measurement of particle spectra, which provide insights into particle production mechanisms. Resonances have very short lifetimes, which are comparable with that of the fireball produced in the collision. Considering that $\mathrm{K}^{*}(892)^{0}$ decays in $\tau_{\mathrm{K}^{* 0}} \sim 4 \mathrm{fm} / c$ and $\phi(1020)$ has a lifetime nearly ten times larger than $\mathrm{K}^{* 0}, \tau_{\phi} \sim 45 \mathrm{fm} / c$, these particles are excellent probes of the hadronic phase of the collision [1]. The yields of resonances can be modified by hadronic scattering processes after chemical freeze-out [2]. The regeneration can increase the measured yield of the intermediate resonance state whereas the re-scattering can decrease the measured yield. The effects of regeneration and re-scattering are expected to be most important for $p_{\mathrm{T}}<2 \mathrm{GeV} / c[2,3]$. The ratios of hadronic resonance yields to the yields of longer-lived hadrons, which are unaffected by these effects, can be used to investigate the re-scattering effects and the chemical freeze-out temperature. The mechanisms which determine the shapes of particle transverse momentum $\left(p_{\mathrm{T}}\right)$ spectra, including the relative strengths of quark recombination $[4,5]$ and hydrodynamical effects $[6,7,8]$, are studied experimentally using many different particle species. The $\phi$, which is a meson with a mass similar to the proton, can provide valuable information regarding the effects of mass and baryon number on the shapes of particle $p_{\mathrm{T}}$ spectra. Measurements in smaller collision systems such as proton-proton (pp) and proton-nucleus ( $\mathrm{p}-\mathrm{A}$ ) provide a necessary baseline for heavy-ion data. In particular, the measurements in $\mathrm{pp}$ are the reference for obtaining the nuclear modification factors $\left(R_{\mathrm{pPb}}, R_{\mathrm{AA}}\right)$, while the $\mathrm{p}-\mathrm{Pb}$ data help to distinguish genuine effects due to the presence of the hot Quark-Gluon Plasma from those related to the presence of a nuclear target beam, also referred to as cold nuclear matter (CNM) effects .

\section{Resonance reconstruction}

This proceeding mainly focuses on results of $\mathrm{K}^{* 0}\left(\left(\mathrm{~K}^{* 0}+\overline{\mathrm{K}}^{* 0}\right) / 2\right)$ and $\phi$ from the ALICE experiment. Further details of these results can be found in $[9,10,11,12,13]$. The detectors which are used for these analyses are presented here with few technical details. The Inner Tracking System (ITS) and Time-Projection Chamber (TPC) are used to reconstruct the primary vertex of the collision and for the tracking in the ALICE central barrel. The Time-of-Flight (ToF) and the TPC are used for particle identification. The V0 scintillator detectors are used to determine centrality and multiplicity classes in $\mathrm{Pb}-\mathrm{Pb}$ [14] and $\mathrm{p}-\mathrm{Pb}$ [15] collisions, respectively, and are also used for trigger selection. The $\mathrm{K}^{* 0}$ and $\phi$ mesons are measured through invariant-mass reconstruction of their identified decay daughters in the charged hadronic decay channels, $\mathrm{K}^{* 0} \rightarrow \mathrm{K}^{+} \pi^{-}\left(\overline{\mathrm{K}}^{* 0} \rightarrow\right.$ $\mathrm{K}^{-} \pi^{+}$) and $\phi \rightarrow \mathrm{K}^{+} \mathrm{K}^{-}$. The measurements in $\mathrm{pp}$ and $\mathrm{Pb}-\mathrm{Pb}$ collisions cover one unit of rapidity $(|y|<0.5)$ in the centre-of-mass reference frame while in $\mathrm{p}-\mathrm{Pb}$ the rapidity range is restricted to $-0.5<y_{\mathrm{cms}}<0$. The combinatorial background is estimated using an event-mixing technique for all collision systems. After subtraction of the combinatorial background, the resonance signals are fitted by using a Breit-Wigner function for $\mathrm{K}^{* 0}$ and convolution of a Breit-Wigner function and a Gaussian for $\phi$. A second-order polynomial is used to describe the residual background for both particles. The raw yields are extracted from the residual background-subtracted resonance 
signal distribution. To measure the invariant spectra, the raw yields are corrected for detector acceptance, efficiency and branching ratio. The $p_{\mathrm{T}}$ spectra are fitted using Levy-Tsallis functions for $\mathrm{pp}$ and $\mathrm{p}-\mathrm{Pb}$ collisions and Boltzmann-Gibbs blast-wave [16] functions for $\mathrm{Pb}-\mathrm{Pb}$ collisions. The $p_{\mathrm{T}}$ integrated particle yields $(\mathrm{d} N / \mathrm{d} y)$ and mean $p_{\mathrm{T}}\left(\left\langle p_{\mathrm{T}}\right\rangle\right)$ of $\mathrm{K}^{* 0}$ and $\phi$ are determined by integrating the $p_{\mathrm{T}}$ spectra in the measured range and by using fit functions to extrapolate the yield in the $p_{\mathrm{T}}$ range where no measurement is available. These quantities have been measured for $\mathrm{Pb}-$ $\mathrm{Pb}$ collisions at $\sqrt{s_{\mathrm{NN}}}=2.76 \mathrm{TeV}$ [13], p-Pb collisions at $\sqrt{s_{\mathrm{NN}}}=5.02 \mathrm{TeV}$ [12], and pp collisions at $\sqrt{s}=7 \mathrm{TeV}[9,10]$.

\section{Results}
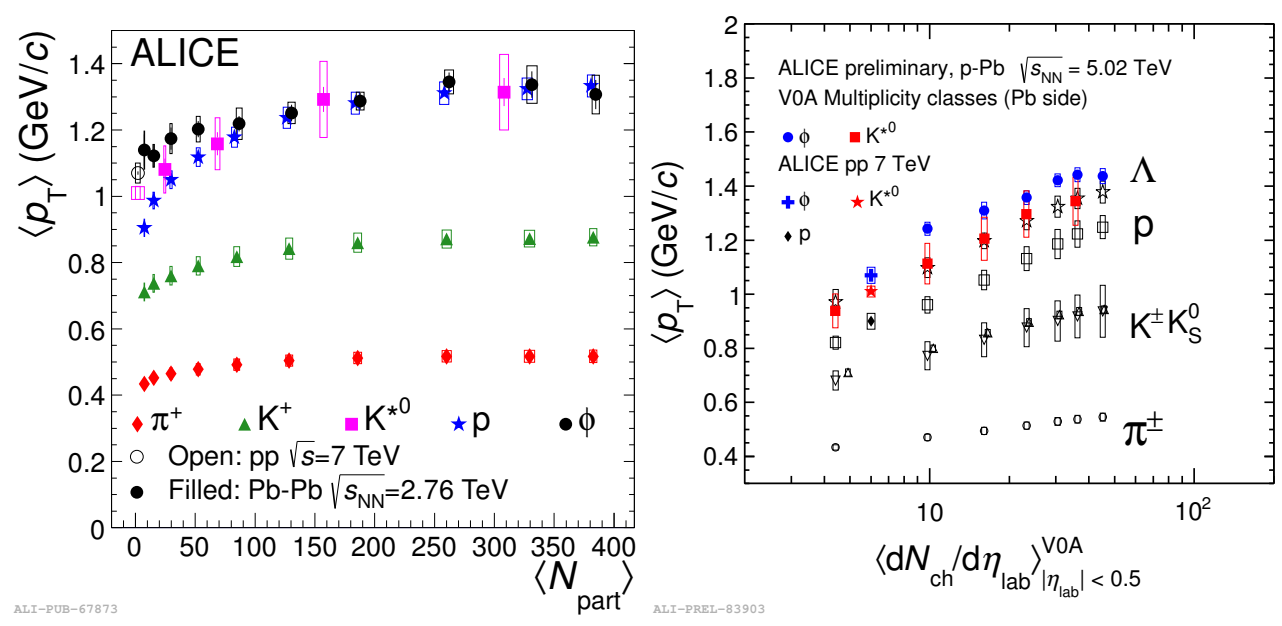

Figure 1: $\left\langle p_{\mathrm{T}}\right\rangle$ of resonances as a function of the average number of participants in the collision in $\mathrm{Pb}-\mathrm{Pb}$ collisions at $\sqrt{s_{N N}}=2.76 \mathrm{TeV}$ [13] (left panel) and as a function of the average charged particle multiplicity density measured in the ALICE central barrel in $\mathrm{p}-\mathrm{Pb}$ collisions at $\sqrt{s_{N N}}=5.02 \mathrm{TeV}$ [11] and pp at $\sqrt{s}=7$ $\mathrm{TeV}$ (right panel). The $\left\langle p_{\mathrm{T}}\right\rangle$ of other hadrons are also compared.

The $\left\langle p_{\mathrm{T}}\right\rangle$ of $\mathrm{K}^{* 0}$ and $\phi$ in various collision systems and comparison to different particles is shown in Fig.1. Left panel of Fig.1 shows the $\left\langle p_{\mathrm{T}}\right\rangle$ of $\mathrm{K}^{* 0}$ and $\phi$ and long-lived hadrons as a function of centrality (average number of participants in the collision) in $\mathrm{Pb}-\mathrm{Pb}$ collisions while right panel reports the $\left\langle p_{\mathrm{T}}\right\rangle$ as a function of the average charged particle multiplicity density in $\mathrm{p}-\mathrm{Pb}$ and pp collisions. It is observed that particles with similar mass, such as $\mathrm{K}^{* 0}, \mathrm{p}$ and $\phi$, have similar $\left\langle p_{\mathrm{T}}\right\rangle$ in the most central $\mathrm{Pb}-\mathrm{Pb}$ collisions. This can be attributed to the hypothesis of particle boost in the hadronic phase being driven by radial flow. The mass-ordering (particles with higher mass are measured to have larger $\left\langle p_{\mathrm{T}}\right\rangle$ ) observed in the most central events for resonances seems to weaken going towards peripheral $\mathrm{Pb}-\mathrm{Pb}$ collisions and in the smaller collision systems. In $\mathrm{p}-\mathrm{Pb}$ collisions, the $\left\langle p_{\mathrm{T}}\right\rangle$ of resonances increases as a function of the average charged particle multiplicity density, as for other hadrons where a weak dependence on centrality in $\mathrm{Pb}-\mathrm{Pb}$ collisions is observed. In $\mathrm{p}-\mathrm{Pb}$ collisions $\left\langle p_{\mathrm{T}}\right\rangle$ of long lived hadrons follows mass ordering, while the $\left\langle p_{\mathrm{T}}\right\rangle$ of $\mathrm{K}^{* 0}$ and $\phi$ is found to be larger than that of protons (the $\left\langle p_{\mathrm{T}}\right\rangle$ of $\phi$ is larger than $\left\langle p_{\mathrm{T}}\right\rangle$ of $\Lambda$ ). A similar trend is also observed in pp collisions at $\sqrt{s}=7 \mathrm{TeV}$. It is also observed that the $\left\langle p_{\mathrm{T}}\right\rangle$ in $\mathrm{p}-\mathrm{Pb}$ shows 

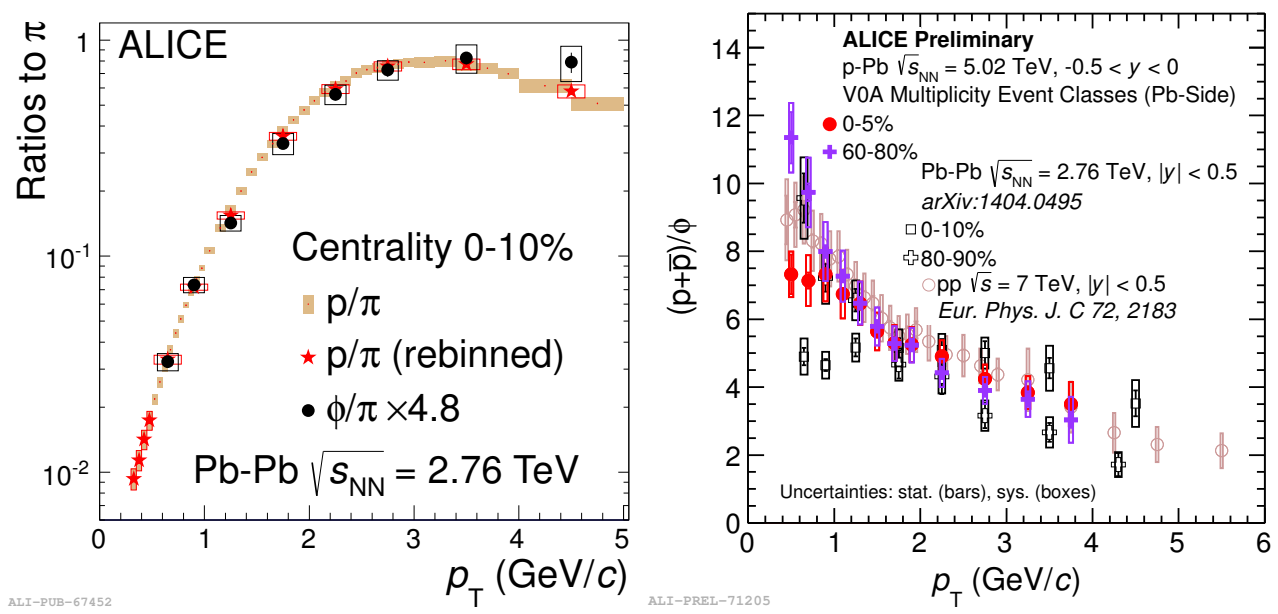

Figure 2: Left panel: $\phi /\left(\pi^{+}+\pi^{-}\right)$compared to the $(\mathrm{p}+\overline{\mathrm{p}}) /\left(\pi^{+}+\pi^{-}\right)$ratio measured in central $(0-10 \%)$ $\mathrm{Pb}-\mathrm{Pb}$ collisions. Right panel: $(\mathrm{p}+\overline{\mathrm{p}}) / \phi$ ratio measured in $\mathrm{p}-\mathrm{Pb}$ in $0-5 \%$ and $60-80 \% \mathrm{~V} 0 \mathrm{~A}$ multiplicity classes, compared to $\mathrm{pp}, 0-10 \%$ and $80-90 \% \mathrm{~Pb}-\mathrm{Pb}$ collisions

a steeper increase with multiplicity than in $\mathrm{Pb}-\mathrm{Pb}$. The left panel of Fig.2 shows the comparison of the $\phi / \pi$ and the $\mathrm{p} / \pi$ ratios in the most central $\mathrm{Pb}-\mathrm{Pb}$ collisions. The similar shape of $\phi / \pi$ and $\mathrm{p} / \pi$ as a function of $p_{\mathrm{T}}$ suggests that the shapes of the $p_{\mathrm{T}}$ distributions of the $\mathrm{p}$ and $\phi$ in this momentum range are determined by the particle masses, which is consistent with the observation of mass ordering of $\left\langle p_{\mathrm{T}}\right\rangle$ in central $\mathrm{Pb}-\mathrm{Pb}$. The $(\mathrm{p}+\overline{\mathrm{p}}) / \phi$ ratio is shown in the right panel of Fig.2 for different centralities in $\mathrm{Pb}-\mathrm{Pb}$, multiplicity classes in $\mathrm{p}-\mathrm{Pb}$ and $\mathrm{pp}$ collisions. This ratio is flat below $4 \mathrm{GeV} / c$ in $0-10 \%$ central $\mathrm{Pb}-\mathrm{Pb}$ collisions. A hint of flattening is also observed for $p_{\mathrm{T}}<$ $1.5 \mathrm{GeV} / c$ in high-multiplicity (0- 5\% V0A multiplicity event class) $\mathrm{p}-\mathrm{Pb}$ collisions. The ratio

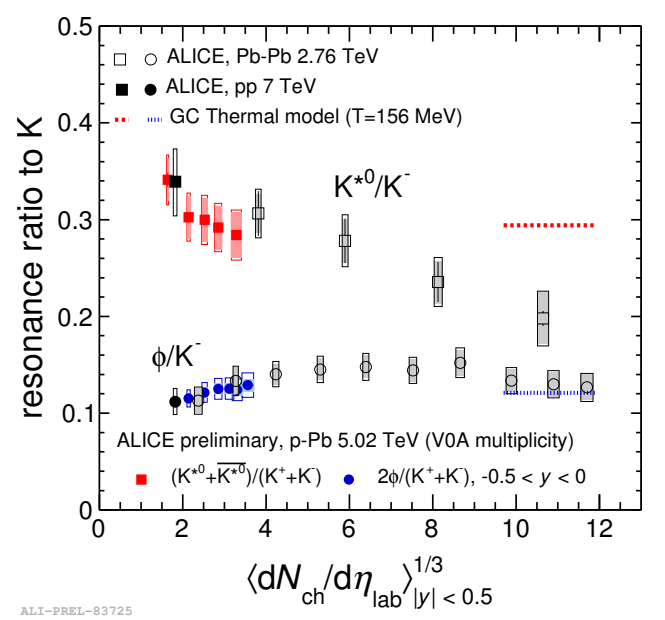

Figure 3: Ratio of resonances to charged kaons measured in $\mathrm{pp}, \mathrm{p}-\mathrm{Pb}$ and $\mathrm{Pb}-\mathrm{Pb}$ collision systems, as a function of the system size. 
in high-multiplicity $\mathrm{p}-\mathrm{Pb}$ is similar to that in $60-80 \%$ peripheral $\mathrm{Pb}-\mathrm{Pb}$ collisions. In peripheral $\mathrm{p}-\mathrm{Pb}$ and $\mathrm{pp}$ collisions the ratio is quantitatively consistent and below $5 \mathrm{GeV} / c$ it decreases steeply with $p_{\mathrm{T}}$, as in peripheral $\mathrm{Pb}-\mathrm{Pb}$ collisions. The ratios $\phi / \mathrm{K}$ and $\mathrm{K}^{* 0} / \mathrm{K}$ measured in $\mathrm{p}-\mathrm{Pb}$ collisions compared to the published measurements for $\mathrm{pp}[10]$ and $\mathrm{Pb}-\mathrm{Pb}$ [13] are shown in Fig.3. The $\phi / \mathrm{K}$ ratio is nearly independent across all systems and multiplicities whereas the $\mathrm{K}^{* 0} / \mathrm{K}$ ratio exhibits a decreasing trend towards more central $\mathrm{Pb}-\mathrm{Pb}$. The ratios are also compared to the values predicted by a grand-canonical thermal model with $\mathrm{T}=156 \mathrm{MeV}$ [17]. The $\phi / \mathrm{K}$ is consistent with the thermal model prediction whereas the $\mathrm{K}^{* 0} / \mathrm{K}$ is about $60 \%$ of the thermal model value in $0-20 \%$ central $\mathrm{Pb}$ $\mathrm{Pb}$ collisions. This can be explained in terms of re-scattering effects, dominating for $p_{\mathrm{T}}<2 \mathrm{GeV} / \mathrm{c}$. An extensive discussion on this can be found in [13].

\section{Summary}

The $\mathrm{K}^{* 0}$ and $\phi$ resonances have been measured in different collision systems at LHC energies. The values of $\left\langle p_{\mathrm{T}}\right\rangle$ in $\mathrm{Pb}-\mathrm{Pb}$ and $\mathrm{p}-\mathrm{Pb}$ collisions follow different trends. The mass ordering of $\left\langle p_{\mathrm{T}}\right\rangle$ values is not observed for $\mathrm{K}^{* 0}, \mathrm{p}, \phi$, and $\Lambda$ in $\mathrm{p}-\mathrm{Pb}$ collisions. The $\phi / \mathrm{p}$ ratio is flat for $p_{\mathrm{T}}<4 \mathrm{GeV} / c$ in central $\mathrm{Pb}-\mathrm{Pb}$ collisions, which is expected from hydrodynamical models since the particles have similar masses. The $\mathrm{K}^{* 0} / \mathrm{K}$ ratio is suppressed in central collisions, consistent with re-scattering of its decay daughters, while the $\phi / \mathrm{K}$ ratio is not suppressed due to the $\phi$ longer lifetime.

\section{References}

[1] Torrieri G and Rafelski J, Phys. Lett. B 509 239, 2001.

[2] Bleicher M and Stocker H, J. Phys. G 30 S111-8, 2004.

[3] Bleicher M and Aichelin J, Phys. Lett. B 530 81-87, 2002.

[4] Fries R, Muller B, Nonaka C and Bass S, Phys. Rev. Lett. 90 202303, 2003.

[5] Fries R, Greco V and Sorensen P, Annu. Rev. Nucl. Part. Sci 58 177-205, 2008.

[6] Qui Z, Shen C and Heinz U, Phys. Lett. B 707 151-5, 2012.

[7] Shen C, Heinz U, Huovinen P and Song H, Phys. Rev. C 84 044903, 2011.

[8] Bozek P and Wyskiel-Piekarska I, Phys. Rev. C 85 064915, 2012.

[9] Aamodt K et al. (ALICE Collaboration), Eur. Phys. J. C 71 1594, 2011.

[10] Abelev B et al. (ALICE Collaboration), Eur. Phys. J. C 72 2183, 2012.

[11] Abelev B et al. (ALICE Collaboration),Phys. Lett. B 728 25-38, 2014.

[12] Bellini F (for the ALICE Collaboration), Nucl. Phys. A 931 846-50, 2014.

[13] Abelev B et al. (ALICE Collaboration), Phys. Rev. C 91, 024609, 2015.

[14] Abelev B et al. (ALICE Collaboration), Phys. Rev. Lett. 106, 032301, 2011.

[15] Toia A (for the ALICE Collaboration), Proceedings of the Quark Matter 2014 conference, Nucl.Phys. A 931, 315-319, 2014.

[16] E. Schnedermann, J. Sollfrank and U. Heinz, Phys. Rev. C 48, 2462, 1993.

[17] Stachel J, Andronic A, Braun-Munzinger P and Redlich K, J. Phys.: Conf. Ser. 509, 012019, 2014. 